\title{
Motor imagery in typing: effects of typing style and action familiarity
}

\author{
Martina Rieger
}

Published online: 5 November 2011

(C) Psychonomic Society, Inc. 2011

\begin{abstract}
The influences of typing style and action familiarity on executed and imagined typing were investigated. A group of touch typists and a group of hunt-and-peck typists were asked to imagine and execute typing texts of different lengths in two different styles: with ten fingers (familiar for touch typists, unfamiliar for hunt-and-peck typists) and with two fingers (unfamiliar for touch typists, familiar for huntand-peck typists). The imagination (but not the execution) of familiar and unfamiliar typing was correlated in both groups, indicating that participants used skill knowledge from the familiar action to imagine the unfamiliar action. Only when touch typists imagined familiar typing accurate motor imagery was observed (similar durations of and positive correlations between imagination and execution). When touch typists imagined unfamiliar typing, the average imagination durations resembled the execution durations, but correlations indicated individual differences in the processes of imagination and execution. Hunt-and-peck typists showed shorter imagination than execution durations with both familiar and unfamiliar typing, indicating that in both styles they did not imagine all details of typing. Also, they did not imagine some
\end{abstract}

\section{Rieger}

Department of Psychology,

Max Planck Institute for Human Cognitive and Brain Sciences, Leipzig, Germany

\section{Rieger}

Department of Psychology, University of Frankfurt, Frankfurt am Main, Germany

\section{Rieger $(\bowtie)$}

M2-Department for Medical Sciences and Management, Institute for Psychology, UMIT-University for Health Sciences, Medical Informatics and Technology,

Eduard Wallnöfer Zentrum 1,

6060 Hall in Tirol, Austria

e-mail: martina.rieger@umit.at details specifically related to unfamiliar typing (reflected in particularly high percentages of absolute error). However, correlations indicated that individual difficulties in executing the unfamiliar action were reflected in the imagination durations. In conclusion, skill knowledge from familiar actions is used to imagine unfamiliar actions. Familiarity with actions promotes accurate motor imagery, but only if stable internal action representations have been acquired, and not if action control relies on online, step-by-step control. However, stable internal action representations of familiar actions may be detrimental for imagery of unfamiliar actions.

Keywords Motor imagery · Typing · Action familiarity Skill

Motor imagery designates movements that are not executed, but instead mentally simulated as if they were (Decety, 1996; Jeannerod, 1994). In behavioral research on motor imagery, the mental chronometry paradigm is often used, in which the durations of imagined actions and executed actions are compared. This comparison relies on the assumption that similar timing of executed and imagined actions reflects similarities in the progress of unfolding actions. Similarities in timing have been reported for a variety of well-known everyday-life actions, such as writing a sentence and drawing a cube (Decety \& Michel, 1989) or walking (Courtine, Papaxanthis, Gentili, \& Pozzo, 2004; Decety, Jeannerod, \& Prablanc, 1989).

However, tasks that entail action requirements only slightly different from their everyday counterparts may affect the timing of executed and imagined actions differentially. For instance, effort applied during executed actions for added weight is not always spontaneously imagined: Imagined walking with $25-\mathrm{kg}$ weight on the shoulders increases mental 
walking duration (Decety et al., 1989), and adding a 2-kg weight in a visual pointing task slows imagined movements down (Cerritelli, Maruff, Wilson, \& Currie, 2000), but in both cases execution durations are unaffected. Less familiar actions may also result in shorter imagination then execution durations, as in movements to awkward and uncommon postures. Here, the complexity and/or familiarity of an action may result in imagery being based on less detailed information (Parsons, 1994).

People perform actions in different ways. For example, experts in typing use ten fingers and touch type, which is the biomechanically most efficient way to type. However, nowadays, many people who type lack formal training in typing and use an idiosyncratic typing style with less than ten fingers (hunt-and-peck typists). They may even be proficient in their style, because they use it on an everyday basis. Nevertheless, skilled touch typists develop typing-specific representations that are not observed in hunt-and-peck typists. In touch typists, the mere perception of a letter on a screen results in automatic activation of the finger that is usually used to type the letter and of the corresponding movement characteristics of the finger (Rieger, 2004). Furthermore, the perception of letter dyads on a screen leads to implicit motor imagery, resulting in a preference for dyads that are biomechanically more comfortable to type (Beilock \& Holt, 2007).

In the present study, the roles of typing style and action familiarity in typing were investigated, using the mental chronometry paradigm. People who type using ten fingers (touch typists) were instructed to execute and imagine tenfinger typing (familiar action) and two-finger typing (unfamiliar action). Furthermore, a group of people who usually type using two fingers (hunt-and-peck typists) were also instructed to execute and imagine two-finger typing (familiar action) and ten-finger typing (unfamiliar action).

When both groups typed in their familiar style, shorter typing durations in touch typists than in hunt-and-peck typists were to be expected, because ten-finger typing is biomechanically more efficient than two-finger typing. Furthermore, in both groups typing durations should be negatively affected by performing an unfamiliar in comparison to a familiar action. Because ten-finger typing is more complex than two-finger typing and requires specific training of the fingers, hunt-andpeck typists should be more negatively affected by the requirement to use ten-finger typing than touch typists would be by the requirement to use two-finger typing. With respect to the relationship between imagination and execution, it was expected that imagination would be accurate (similar durations of imagination and execution, positive correlations between imagination and execution) when participants imagined typing in their familiar typing style. However, hunt-and-peck typists should have difficulties imagining unfamiliar ten-finger typing (duration differences between imagination and execution, no or low correlations between imagination and execution), because ten-finger typing is more complex than their familiar typing style. The expectations concerning unfamiliar twofinger typing in touch typists were not specific. On the one hand, touch typists have the required finger skills to perform an action that is similar to but less complex than ten-finger typing accurately, and they should be able to use this motor knowledge in motor imagery. On the other hand, two-finger typing may require touch typists to inhibit typing-specific representations that they have acquired (Beilock \& Holt, 2007; Rieger, 2004), which may be more difficult in imagination than in execution, because during imagination participants need to rely on their internal representations, whereas during execution online feedback may support executing an unfamiliar action.

\section{Method}

Participants

Touch typists ( $n=18,7$ male, 11 female; age: $M=25.9$ years, $S D=6.2$ ) were able to type fluently using ten-finger typing. Hunt-and-peck typists $(n=18,9$ male, 9 female; age: $M=$ 25.2 years, $S D=3.8$ ) usually used two-finger typing. All of the participants were right-handed, had normal or correctedto-normal vision, and were paid to participate in the experiment.

\section{Task and procedure}

Participants were asked to type three short texts taken from a magazine (which required 84, 149, and 248 keystrokes, including spaces). In the execution conditions, participants typed the text into a text editor on a personal computer. In the imagination conditions, participants pressed the space bar when they started and when they finished imagining typing a text. The KBLog program (Chang, Wang, Luh, \& Hwang, 2004) registered the computer system time and the identity of every keystroke.

Upon arrival, participants were asked to read the texts until they felt familiar with them. Participants then typed the three texts in each of the experimental conditions. No restrictions were imposed on the visibility of the keyboard or the screen. Participants performed four experimental conditions: imagined ten-finger typing (Imagination10), executed ten-finger typing (Execution10), imagined twofinger typing (Imagination02), and executed two-finger typing (Execution02). Before ten-finger typing, hunt-andpeck typists were shown a picture of the keyboard depicting the finger assignments of ten-finger typing and were given a detailed verbal explanation. In the imagination conditions, 
participants were asked to imagine every keystroke, how it feels to perform the movements, and how it feels to press the keys. They were also asked to put their hands flat on the table, which was observed by the experimenter. Participants were told not to correct errors in the execution conditions. The order of familiarity (unfamiliar or familiar action) was blocked and counterbalanced between participants. The order of action (imagination or execution) was also counterbalanced between participants; in both familiarity conditions, the action order was the same.

\section{Data analyses}

In the execution conditions, the typing durations of each text were calculated using the difference between the first and last keystrokes. In the imagination conditions, durations were calculated using the difference between pressing the space bar at the beginning and at the end of a text. From this difference the average duration of one keystroke, calculated from the execution conditions (separately for the two typing styles), was subtracted twice, to account for pressing the space bar at the beginning and the end of each text. An ANOVA with the between-participants factor group (touch or hunt-and-peck typists) and the withinparticipants factors familiarity (familiar or unfamiliar action), action (imagination or execution), and text (84, 149 , or 248 keystrokes) was performed on typing durations.

In order to analyze the absolute deviations of imagination from execution, irrespective of typing duration, the percentage of absolute error was calculated (Munzert, 2008). The ANOVA on percentages of absolute errors was performed without the action factor.

For each group, correlations between the different action conditions were calculated separately for each text and overall, because positive correlations across tasks indicate that timing performance is not task specific, but rather that common timing mechanisms are effective (Zelaznik, Spencer, Ivry, Baria, Bloom, Dolansky, \& Whetter, 2005). Furthermore, correlations between the number of errors and the differences between imagination and execution were calculated, because differences between imagination and execution are related to errors and error corrections in typing (Rieger, Martinez, \& Wenke, 2011). Correlations were compared using Fisher's $z$ test.

\section{Results}

Imagined and executed typing duration

In the ANOVA on typing durations (see Fig. 1a), all main effects and interactions were significant. The significant

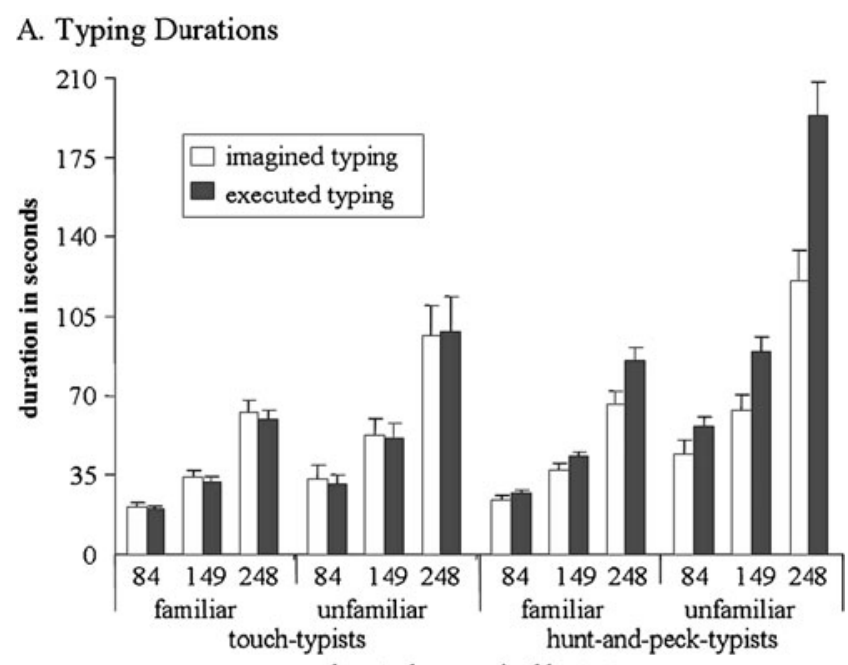

keystrokes required by text

\section{B. \% Absolute Error}

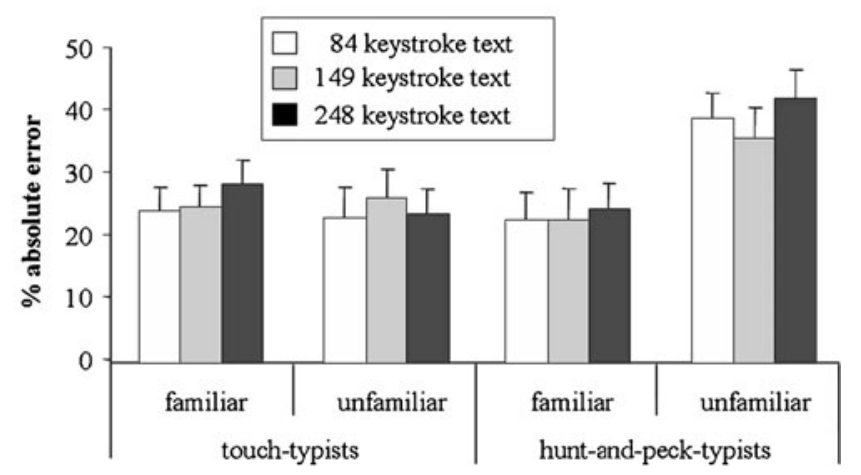

Fig. 1 Means and standard errors of (a) typing durations and (b) percentages of absolute error

main effect of text, $F(2,68)=296.01, p<.001, \eta_{\mathrm{p}}^{2}=.9$, indicated that typing durations increased with longer texts (all $p \mathrm{~s}<.05$ ). The significant main effect of group, $F(1,34)=10.85, p=.002, \eta_{\mathrm{p}}^{2}=.24$, indicated that huntand-peck typists $(M=70 \mathrm{~s})$ had longer typing durations than touch typists $(M=49 \mathrm{~s})$. The significant main effect of familiarity, $F(1,34)=43.88, p<.001, \eta_{\mathrm{p}}^{2}=.56$, indicated that both groups had shorter typing durations with the familiar than with the unfamiliar action. Touch typists also had longer typing durations than hunt-and-peck typists when they used two-finger typing $(p<.05)$.

The interaction between group and familiarity, $F(1,34)=$ 5.61, $p=.024, \eta_{\mathrm{p}}^{2}=.14$, indicated that the difference between using the familiar versus the unfamiliar action was greater in hunt-and-peck typists (difference: $M=47 \mathrm{~s}$ ) than in touch typists (difference: $M=22 \mathrm{~s}, p<.05$ ). The interactions between familiarity and text, $F(2,68)=36.98, p<.001$, $\eta_{\mathrm{p}}^{2}=.53$, group and text, $F(2,68)=12.55, p=.001$, $\eta_{\mathrm{p}}^{2}=.27$, and group, familiarity, and text, $F(2,68)=5.9$, 
$p=.01, \eta_{\mathrm{p}}^{2}=.15$, indicated that group differences increased with text length, that the differences between familiar and unfamiliar typing increased with text length, and that the familiar-unfamiliar differences with text length were more pronounced in hunt-and-peck than in touch typists.

In order to explain all of the effects in which action was involved as a factor, separate ANOVAs were conducted for each group with the factors familiarity, action, and text. Action had no effect on typing durations in touch typists (all $F_{\mathrm{s}} \leq 1.35$ ). In contrast, the ANOVA for hunt-and-peck typists showed a significant main effect of action, $F(1,17)=$ $40.12, p<.001, \eta_{\mathrm{p}}^{2}=.7$, indicating that execution $(M=82 \mathrm{~s})$ took longer than imagination $(M=59 \mathrm{~s})$. This was qualified by a significant interaction between action and text, $F(2,34)=$ $39.1, p<.001, \eta_{\mathrm{p}}^{2}=.7$, which indicated that the executionimagination differences increased with text length (all $p s<.05)$ and that imagination and execution did not differ significantly from each other for the shortest text $(p>.05)$. This corresponds to results showing that differences between imagination and execution increase with longer movement durations (Grealy \& Shearer, 2008). A significant interaction between action and familiarity, $F(1,17)=17.16, p<.001$, $\eta_{\mathrm{p}}^{2}=.5$, indicated that the execution-imagination difference was higher for the unfamiliar (difference: $M=37 \mathrm{~s}$ ) than for the familiar (difference: $M=10 \mathrm{~s}, p<.05$ ) action.

Percentages of absolute error

The ANOVA on percentages of absolute error (see Fig. 1b) showed a significant main effect of familiarity, $F(1,34)=$ 4.9, $p=.034, \eta_{\mathrm{p}}^{2}=.13$, which was qualified by a significant interaction between group and familiarity, $F(1$, $34)=7.34, p=.01, \eta_{\mathrm{p}}^{2}=.19$. There were no significant differences between familiar typing in touch typists $(M=26 \%)$, unfamiliar typing in touch typists $(M=24 \%)$, and familiar typing in hunt-and-peck typists $(M=24 \%$, all $p$ s > .05). However, the percentage of absolute error was higher for unfamiliar typing in hunt-and-peck typists $(M=39 \%)$ than for all other conditions (all $p \mathrm{~s}<.05$ ). None of the other main effects or interactions were significant.

\section{Correlations}

Significant positive correlations (see Table 1) between imagination and execution were observed in both groups when participants used their familiar typing style. When participants used the unfamiliar typing style, again positive and significant correlations were observed in hunt-and-peck typists, but not in touch typists. The overall correlation between imagination and execution in the unfamiliar twofinger-typing condition for touch typists was significantly lower than the correlations in the familiar ten-finger-typing condition for the same group $(p<.001)$, the familiar twofinger-typing condition for hunt-and-peck typists $(p<.05)$, and the unfamiliar ten-finger-typing condition for hunt-andpeck typists $(p<.001)$. This result cannot be explained by reduced variability in the unfamiliar typing condition among touch typists, because the standard deviations of executed typing were similar for ten-finger typing in touch typists $(S D=30)$, two-finger typing in touch typists $(S D=$ $28)$, and two-finger typing in hunt-and-peck typists $(S D=$ $25)$. However, the high variability of executed typing might partly explain the particularly high correlation for unfamiliar typing in hunt-and-peck typists $(S D=147)$.

Execuction 10 was not significantly correlated with Execution02 in either group. Imagination 10 and Imagination 02 , however, were significantly correlated in both groups. Statistically, the difference between the two correlations did not reach significance in touch typists $(p=.19)$, but it did in hunt-and-peck typists $(p=.04)$.

In each group, only one of the correlations between the execution-imagination difference and the number of errors was significant. This is consistent with results indicating that in general, corrected but not uncorrected errors are related to differences between imagination and execution in typing (Rieger et al., 2011).

\section{Discussion}

In the present study, the effects of usual typing style and action familiarity on the similarities and differences between action imagination and action execution were investigated. Touch typists showed accurate timing of imagined typing, regardless of action familiarity. However, significant positive correlations between imagination and execution were only observed in familiar typing, not in unfamiliar typing. In contrast, hunt-and-peck typists showed shorter imagination than execution durations in familiar and unfamiliar typing. They were particularly inaccurate when imagining the unfamiliar typing style (percentages of absolute error). Nevertheless, imagination and execution were correlated for both familiar and unfamiliar typing. In both groups, execution of familiar and unfamiliar typing was not correlated, but imagination of familiar and unfamiliar typing was.

The observation that execution of familiar and execution of unfamiliar typing were not correlated indicates that different skills are required in the two typing styles. The correlations between imagination of familiar and imagination of unfamiliar typing most likely indicate that participants relied on motor knowledge from the familiar action when they imagined the unfamiliar action. The reverse scenario seems unlikely. It is possible that some typing- 
Table 1 Correlations

Significance testing of correlations was performed one sided; duration difference $=$ duration difference between imagination and execution. ${ }^{*} p<.05 .{ }^{* *} p<.01$

\begin{tabular}{lllll}
\hline & \multicolumn{2}{l}{ Keystrokes per Text } & \\
\cline { 2 - 4 } & 84 & 149 & 248 & Overall \\
\hline Touch Typists & & & & \\
Familiar: Imagination10 $\times$ Execution10 & $.65^{* *}$ & $.73^{* *}$ & $.62^{* *}$ & $.7^{* *}$ \\
Unfamiliar: Imagination02 $\times$ Execution02 & .14 & -.01 & -.02 & .001 \\
Execution10 $\times$ Execution02 & .30 & .31 & .26 & .33 \\
Imagination10 $\times$ Imagination02 & $.47^{*}$ & $.50^{*}$ & $.61^{* *}$ & $.58^{* *}$ \\
Familiar: \# errors $\times$ Duration difference & -.05 & -.02 & $.45^{*}$ & .26 \\
Unfamiliar: \# errors $\times$ Duration difference & -.15 & -.06 & -.02 & -.09 \\
Hunt-and-Peck Typists & & & & \\
Familiar: Imagination02 $\times$ Execution02 & $.41^{*}$ & $.46^{*}$ & $.57^{* *}$ & $.58^{* *}$ \\
Unfamiliar: Imagination10 $\times$ Execution10 & $.78^{* *}$ & $.80^{* *}$ & $.82^{* *}$ & $.84^{* *}$ \\
Execution10 $\times$ Execution02 & .25 & .19 & .03 & .13 \\
Imagination10 $\times$ Imagination02 & $.50^{*}$ & $.49^{*}$ & $.67^{* *}$ & $.64^{* *}$ \\
Familiar: \# errors $\times$ Duration difference & -.13 & $.52^{*}$ & .15 & .37 \\
Unfamiliar: \# errors $\times$ Duration difference & -.1 & .16 & .39 & .25 \\
\hline
\end{tabular}

unspecific "imagination factor" influenced the imagination of both familiar and unfamiliar typing. Such a typingunrelated factor is, however, difficult to conceive.

Motor imagery is thought to rely on memories (Annett, 1996) or (from a computational viewpoint) on internal models of actions (Davidson \& Wolpert, 2005). If the action is familiar, established internal models can be used for motor imagery. Imagining unfamiliar actions may be similar to executing them: Initial learning of new actions is assumed to rely on internal models for other, similar, known actions. Only after acquisition of the new action, the specific internal models most suitable for it are activated and/or newly learned internal models can be used (Imamizu, Higuchi, Toda, \& Kawato, 2007; Wolpert \& Kawato, 1998). Thus, internal models for the familiar typing style may be used to imagine the unfamiliar typing style.

The expectation that imagination would be accurate for familiar typing in both groups was only partly met. Touch typists showed accurate imagination of the familiar typing style (similar durations of and significant positive correlations between imagination and execution). However, huntand-peck typists showed shorter imagination than execution durations in familiar (and in unfamiliar) typing. Shorter imagination than execution durations might indicate that imagery is based on less detailed information (Parsons, 1994). Thus, hunt-and-peck typists might not imagine all aspects of executed typing even in the familiar typing style. Shorter durations of imagination than execution can also be seen as reflecting a learning stage in which detailed skill knowledge has not yet been established (Guillot \& Collet, 2005; Reed, 2002). Hunt-and-peck typists, who use a very inefficient (two-finger) way of typing, may therefore be regarded as nonexperts, even though they show some proficiency in typing in their own style, since they have shorter durations in two-finger typing than touch typists.

Not much is known about how hunt-and peck typists represent the task of typing, apart from that they do not have some typing-related representations that touch typists have (Beilock \& Holt, 2007; Rieger, 2004). Nevertheless, one may speculate that due to the nature of two-finger typing, spatial representations of the keyboard may be more important than kinesthetic information from the fingers, as motor information is not very specific, and thus not informative. However, the keyboard was visible to participants in all conditions, rendering it likely that participants used the available information to update spatial representations.

Furthermore, motor imagery might require a shift in attention from external to internal stimuli (Munzert, 2008), and internal aspects (i.e., kinesthesis) were also emphasized in the instructions. Hunt-and-peck typists may not accurately represent those internal aspects, which may not be an important aspect of their typing-related representations. Consequently they do not sufficiently imagine these internal aspects.

A related explanation is that hunt-and-peck typists rely on explicit online step-by-step control during executed typing, and are thus dependent on feedback. The lack of feedback from the environment during imagery can negatively affect accuracy (Campos, Siegle, Mohler, Bülthoff, \& Loomis, 2009). Thus, if hunt-and-peck typists rely on feedback from the environment during execution, certain aspects of typing might not be taken into account in imagination.

Both groups did not accurately imagine unfamiliar actions; this was reflected in their data patterns in different ways. In touch typists, even though there were no differences in average durations, imagination and execution were 
not correlated in the unfamiliar two-finger typing action. The dissociation between durations and correlations indicates that similarities in (average) timing do not necessarily imply similarities in processes: The lack of significant correlations points to individual differences in the processes of imagination and execution (Zelaznik et al., 2005). These individual differences most likely result from differences in imagining the unfamiliar action, because during imagination participants need to rely on their internal representations, whereas during execution online feedback may support executing unfamiliar actions.

First, touch typists may have had varying degrees of experience in typing with fewer than ten fingers. Participants with experience in two-finger typing might have used internal models for two-finger typing, instead of internal models for 10-finger-typing to imagine the unfamiliar action. However, imagination of familiar and of unfamiliar actions was positively correlated (and the correlation was not different from the correlation in huntand-peck typists), rendering this explanation unlikely.

Second, in touch typists, effector-dependent representations, reflecting kinesthetic and tactile sensations, are more pronounced than spatial representations (Rieger, 2004). Skilled typists even have difficulties imagining precise locations on the keyboard (Liu, Crump, \& Logan, 2010). Variability in spatial representations might have affected imagination of two-finger typing. However, the keyboard was visible in all conditions, and could thus be used for spatial updating.

Third, well-learned skills such as typing are believed to be based on automated control structures that are largely outside of explicit attentional control, and attention to such automatic processes disturbs performance (Beilock, Carr, MacMahon, \& Starkes, 2002; Logan \& Crump, 2009). The requirement to imagine an unfamiliar style might have caused touch typists to pay attention to automated, usually unattended aspects of typing, which might have been more difficult for some participants.

Fourth, when seeing a letter, automatic activation of the fingers (Rieger, 2004) and automatic motor imagery (Beilock \& Holt, 2007) occurs in touch typists. Such automatic processes might interfere with motor imagery of the unfamiliar action, and this might be more pronounced in and/or more difficult to ignore for some participants.

As in familiar typing, hunt-and-peck-typists showed shorter imagination than execution durations in unfamiliar typing. Again, specific aspects of executed actions might not be imagined due to the lack of detailed action representations (Guillot \& Collet, 2005; Parsons, 1994; Reed, 2002) and/or the lack of online feedback (Campos et al., 2009). Crucially, the percentage of absolute error was particularly high in this condition. This indicates that certain aspects specifically related to ten-finger typing might not be accurately imagined, and further indicates that imagining the unfamiliar typing style might have been much more difficult for hunt-and-peck than touch typists.

Nevertheless, in hunt-and-peck typists a positive correlation between imagination and execution was observed in unfamiliar typing, indicating that participants who took longer to execute ten-finger typing also took longer to imagine ten-finger typing. Thus, individual difficulties in executing the unfamiliar, more complex action are reflected in the imagination durations.

In conclusion, skill knowledge from familiar actions is used to imagine unfamiliar actions. Whether familiarity with an action promotes accurate motor imagery depends on the type of action representations that have been acquired. Stable internal action representations and less reliance on online step-by-step control may be predictors for accurate imagery of familiar actions. However, stable internal action representations may also negatively affect motor imagery of similar but unfamiliar actions. These results have implications for mental training of skills, in which motor imagery is assumed to be one of the mechanisms contributing to its effectiveness: The type of action representations acquired might affect the training's outcome.

Author note The data for this study were collected at the Max Planck Institute for Human Cognitive and Brain Sciences, Leipzig. I thank Roman Schoenitz, Silke Meissner, and Gudrun Henze for help with data collection and the analysis.

\section{References}

Annett, J. (1996). On knowing how to do things: A theory of motor imagery. Cognitive Brain Research, 3, 65-69.

Beilock, S. L., Carr, T. H., MacMahon, C., \& Starkes, J. L. (2002). When paying attention becomes counterproductive: Impact of divided versus skill-focused attention on novice and experienced performance of sensorimotor skills. Journal of Experimental Psychology: Applied, 8, 6-16.

Beilock, S. L., \& Holt, L. E. (2007). Embodied preference judgments: Can likeability be driven by the motor system? Psychological Science, 18, 51-57. doi:10.1111/j.1467-9280.2007.01848.x

Campos, J. L., Siegle, J. H., Mohler, B. J., Bülthoff, H. H., \& Loomis, J. M. (2009). Imagined self-motion differs from perceived selfmotion: Evidence from a novel continuous pointing method. PLoS ONE, 4(11), e7793. doi:10.1371/journal.pone.0007793

Cerritelli, B., Maruff, P., Wilson, P., \& Currie, J. (2000). The effect of an external load on the force and timing components of mentally represented action. Behavioural Brain Research, 108, 91-96.

Chang, C.-H., Wang, J.-D., Luh, J.-J., \& Hwang, Y.-H. (2004). Development of a monitoring system for keyboard users' performance. Ergonomics, 47, 1571-1581. doi:10.1080/ 00140130412331290871

Courtine, G., Papaxanthis, C., Gentili, R., \& Pozzo, T. (2004). Gaitdependent motor memory facilitation in covert movement execution. Cognitive Brain Research, 22, 67-75.

Davidson, P. R., \& Wolpert, D. M. (2005). Widespread access to predictive models in the motor system: A short review. Journal of Neural Engineering, 2, S313-S319.

Decety, J. (1996). The neurophysiological basis of motor imagery. Behavioural Brain Research, 77, 45-52. 
Decety, J., Jeannerod, M., \& Prablanc, C. (1989). The timing of mentally represented actions. Behavioural Brain Research, 34, 35-42.

Decety, J., \& Michel, F. (1989). Comparative analysis of actual and mental movement times in two graphic tasks. Brain and Cognition, 11, 87-97. doi:10.1016/0278-2626(89)90007-9

Grealy, M. A., \& Shearer, G. F. (2008). Timing processes in motor imagery. European Journal of Cognitive Psychology, 20, 867892. doi: $10.1080 / 09541440701618782$

Guillot, A., \& Collet, C. (2005). Duration of mentally simulated movement: A review. Journal of Motor Behavior, 37, 10-20. doi:10.3200/JMBR.37.1.10-20

Imamizu, H., Higuchi, S., Toda, A., \& Kawato, M. (2007). Reorganization of brain activity for multiple internal models after short but intensive training. Cortex, 43, 338-349.

Jeannerod, M. (1994). The representing brain: Neural correlates of motor intention and imagery. The Behavioral and Brain Sciences, 17, 187-245. doi:10.1017/S0140525X00034026

Liu, X., Crump, M. J. C., \& Logan, G. D. (2010). Do you know where your fingers have been? Explicit knowledge of the spatial layout of the keyboard in skilled typists. Memory \& Cognition, 38, 474484. doi:10.3758/MC.38.4.474

Logan, G. D., \& Crump, M. J. C. (2009). The left hand doesn't know what the right hand is doing: The disruptive effects of attention to the hands in skilled typewriting. Psychological Science, 20, 1296-1300. doi:10.1111/j.1467-9280.2009.02442.x
Munzert, J. (2008). Does Level of expertise influence imagined durations in open skills? Played versus imagined durations of badminton sequences. International Journal of Sport and Exercise Psychology, 6, 24-38.

Parsons, L. M. (1994). Temporal and kinematic properties of motor behavior reflected in mentally simulated action. Journal of Experimental Psychology: Human Perception and Performance, 20, 709-730. doi:10.1037/0096-1523.20.4.709

Reed, C. L. (2002). Chronometric comparisons of imagery to action: Visualizing versus physically performing springboard dives. Memory \& Cognition, 30, 1169-1178. doi:10.3758/ BF03213400

Rieger, M. (2004). Automatic keypress activation in skilled typing. Journal of Experimental Psychology: Human Perception and Performance, 30, 555-565. doi:10.1037/0096-1523.30.3.555

Rieger, M., Martinez, F., \& Wenke, D. (2011). Imagery of errors in typing. Cognition, 121, 163-175. doi:10.1016/j.cognition.2011.07.005

Wolpert, D. M., \& Kawato, M. (1998). Multiple paired forward and inverse models for motor control. Neural Networks, 11, 13171329. doi:10.1016/S0893-6080(98)00066-5

Zelaznik, H. N., Spencer, R. M. C., Ivry, R. B., Baria, A., Bloom, M., Dolansky, L., ...Whetter, E. (2005). Timing variability in circle drawing and tapping: Probing the relationship between event and emergent timing. Journal of Motor Behavior, 37, 395-403. doi:10.3200/JMBR.37.5.395-403 